\title{
Relación entre las estrategias de enseñanza y el rendimiento académico en los estudiantes de Primaria y EBI de la Universidad Nacional «Santiago Antúnez de Mayolo»
}

\author{
Relationship of theaching strategies and the academic achievement in \\ Primary and EBI students of the «Santiago Antúnez de Mayolo» \\ National University \\ Laura Nivin Vargas ${ }^{1}$, Víctor Zegarra Chauca ${ }^{1}$ y César Brito Mallqui ${ }^{1}$
}

\section{RESUMEN}

La investigación da cuenta de la relación que existe entre las estrategias de enseñanza y el rendimiento académico en los estudiantes de la especialidad de Primaria y EBI de la Facultad de Ciencias Sociales, Educación y de la Comunicación de la UNASAM. Ante los avances tecnológicos, así como de la pedagogía moderna y su implementación en el sistema universitario nos propusimos describir, analizar y determinar el tipo y nivel de relación que existe entre las estrategias de enseñanza que utilizan los docentes al desarrollar sus sesiones de clase y el nivel de rendimiento y satisfacción por parte de los estudiantes de la referida especialidad. El estudio corresponde al tipo correlacional. Se realizó con estudiantes de la especialidad de Primaria y EBI, eligiendo a 131 de ellos como muestra. Para el análisis de los datos provenientes de las encuestas realizadas, desde una perspectiva cuantitativa, se recurrió al uso de la estadística descriptiva y del formato SPSS. Los resultados revelan que las estrategias de enseñanza guardan una relación positiva con el rendimiento académico de los estudiantes.

Palabras clave: estrategias de enseñanza; rendimiento académico; correlación.

\begin{abstract}
This research realizes the relationship between teaching strategies and academic achievement in students of primary and specialty EBI Faculty of Social Sciences and Education Communication UNASAM. Given technological advances, as well as modern education and its implementation in the university system we set out to describe, analyze and determine the type and level of relationship between the teaching strategies used by teachers to develop their classroom sessions and level of performance and satisfaction of students of that specialty.
\end{abstract}

1 Universidad Nacional «Santiago Antúnez de Mayolo». Huaraz, Perú. 
The research is for the correlational. The study was conducted with students of primary and specialty EBI whose sample consisted of 131 students corresponding to the most advanced semesters. The analysis of data from surveys of students in Primary and EBI was conducted from a quantitative perspective by using descriptive statistics using SPSS format. The analysis of the data reveals that teaching strategies are positively related to academic performance of students.

Keywords: teaching strategies; academic performance; correlation.

\section{ICHIKLLACHAW}

Kay yachay ashiychaw tarikashqa maa UNASAMPA yachatsikuqpaq yachatsikuychaw, ishkay shimichawwan yuyaychaw (EBI) yachakuq wamrakunapa imanaw imanawpis yachatsikuywan yachakuqkunapa yachaynin tanqanakushqantam. Yachatsikuykuna kanan miraykan, tsay rurinchawmi ripakayta munayarquu, maa imanawshi yachatsikuqkuna uryayashqan yachakuqkunapa yachayninwan kawayan, niykur tsaypaq kay yachakuqkuna kushukuyanku, manaku. Kay urya kashqa kay tumaqkuna imanawpis tanqanakuyanqanta ashirmi; tsaypaq rikapaayarquu huk pachak kima chunka huk yachakuqkunatam. Niykur tapukuykunapita yaski yarqamuqkunaqam rikapatsiyarquu SPSSwan. Yarqamushqankunam willakun kay ishkay tumaqkunam yachakuqkunapa allinpaqmi tanqakankuyan.

Pushaq shimikuna: Imanawpis yachatsikuy; alli yachay; yachakuqpa tinkunankuna.

\section{INTRODUCCIÓN}

En la educación universitaria, el docente cumple una función básica: enseñar; este proceso consiste en crear un clima de confianza, sumamente motivador; a la vez, proveer los medios y materiales necesarios que permitan a los estudiantes aprender y desarrollar sus potencialidades. La enseñanza se concreta en el desarrollo de los procesos cognitivos. Para tal fin, el docente hace uso de un conjunto de estrategias dentro de las cuales precisa y comparte experiencias conceptuales, procedimentales y actitudinales con el fin de posibilitar aprendizajes significativos en los discentes.

La docencia es, entonces, una tarea esencial de la universidad; pero no de cualquier tipo, sino de alta especialización. Esto se corrobora en el nivel de formación académica y profesional que alcanza y en la función que cumple. En tal virtud, la docencia universitaria, requiere de profesionales que transiten por el proceso de aprender a enseñar a fin de realizar el acto educativo acorde con las exigencias y niveles del mundo globalizado. Ello implica, de antemano, informar al estudiante sobre los objetivos o competencias a alcanzar y la selección de procedimientos a implementar con sus respectivas justificaciones de valor, a fin de que progresivamente se vayan formando como aprendices estratégicos. Para eso, requiere hacer una selección consciente y cuidadosa de estrategias que le permitan desarrollar su labor con eficacia y eficiencia para el logro de los propósitos deseados.

Esta investigación comienza analizando lo que ha sido la teoría y la práctica de la enseñanza tomando como referencia los planteamientos de Mayer (1984, 2004), Eggen 
y Kauchak (1999), Castellá et al. (2007), Mercer (2001), entre otros, y los recursos o medios validados por su utilidad en el campo de la investigación psicoeducativa, que nos ofrece Díaz-Barriga y Hernández (2010) para precisar si tienen incidencia o relación con el rendimiento académico de los estudiantes de la especialidad de Primaria y Educación Bilingüe Intercultural (EBI) de la Facultad de Ciencias Sociales, Educación y de la Comunicación de la Universidad Nacional «Santiago Antúnez de Mayolo» (UNASAM).

La presente investigación aporta a la comunidad universitaria datos sobre dos problemas manifiestos en el sistema universitario: las estrategias de enseñanza y el rendimiento académico, para realizar propuestas pertinentes. El objetivo del trabajo de investigación consistió en analizar la relación que existe entre las estrategias de enseñanza y el rendimiento académico en los estudiantes de la especialidad de Primaria y EBI de la Facultad de Ciencias Sociales, Educación y de la Comunicación de la UNASAM.

\section{MATERIAL Y MÉTODOS}

El estudio realizado fue de tipo correlacional, pues buscaba conocer la relación que existe entre las estrategias de enseñanza y el rendimiento académico de los estudiantes de la especialidad de Primaria y EBI de la Facultad de Ciencias Sociales, Educación y de la Comunicación de la UNASAM. Diseño no experimental de tipo transeccional correlacional. La población estuvo conformada por la totalidad de los estudiantes de la especialidad de Primaria y EBI de la Facultad de Ciencias Sociales, Educación y de la Comunicación de la UNASAM. En total fueron 131, de los diez semestres académicos. La muestra fue de tipo censal porque estuvo compuesta por la totalidad de estudiantes de la especialidad de primaria EBI incluyendo todos los semestres académicos.

La técnica que se utilizó para la recopilación de información fue la encuesta, y mediante el instrumento cuestionario se obtuvo información de la muestra, respecto a la dimensión teórica de la variable. Pero, previo a ello, se realizó un plan piloto con el fin de precisar adecuadamente la estructura de la técnica que posibilite obtener información válida y confiable. La observación de campo, a través del instrumento ficha de observación se recabó información acerca de la dimensión práctica de la variable que nos permitió establecer qué tan notoria es la problemática descrita. Estuvo conformada por ítems, los cuales fueron elaborados y aplicados por los investigadores en la fecha y tiempo establecido.

Luego, se procedió a la revisión, tabulación, análisis e interpretación y los resultados expresados en porcentajes, cuadros y gráficos; para, finalmente, arribar a conclusiones y expresar las recomendaciones pertinentes. Se hizo uso de la estadística descriptiva e inferencial, con la ayuda del paquete estadístico SPSS V.20.

\section{RESULTADOS}

Los resultados reflejan la relación manifiesta entre las estrategias de enseñanza y el rendimiento académico en estudiantes de la escuela de Educación de acuerdo a lo que 
arrojó la prueba estadística Correlación de Spearman. En ella se podrá percibir cuál de las estrategias dadas a conocer se relaciona con mayor amplitud con el rendimiento académico.

Tabla 1. Prueba de la Correlación de Spearman entre las estrategias de enseñanza y el rendimiento académico

\begin{tabular}{|c|c|c|}
\hline & Indicador & Rendimiento académico \\
\hline \multirow{3}{*}{ Las estrategias de enseñanza } & Coeficiente de correlación & .671 \\
\hline & Sig. (bilateral) & .000 \\
\hline & $\mathrm{N}$ & 131 \\
\hline
\end{tabular}

En la tabla 1 se evidencia la correlación entre las variables: estrategias de enseñanza y rendimiento académico. Se percibe en las cantidades que muestran después de aplicada la prueba estadística donde el coeficiente de correlación es de .671, signo bilateral .000, lo cual implica el carácter aceptable entre ambas variables.

Tabla 2. Prueba de la Correlación de Spearman entre las estrategias para activar y usar los conocimientos previos y el rendimiento académico

\begin{tabular}{llc}
\hline & Indicador & Rendimiento académico \\
\hline Estrategias para activar y & Coeficiente de correlación & .591 \\
usar los conocimientos & Sig. (bilateral) & .000 \\
previos & $\mathrm{N}$ & 131 \\
\hline
\end{tabular}

En la tabla 2 se muestra la correlación entre las variables: estrategias para activar y usar los conocimientos previos y el rendimiento académico dadas a conocer en la hipótesis específica. Se observa en las cantidades que muestran después de aplicada la prueba estadística; donde el coeficiente de correlación arroja la cantidad de .591, signo bilateral .000 , lo cual implica el carácter positivo entre ambas variables.

Tabla 3. Prueba de la Correlación de Spearman entre las estrategias para mejorar la integración constructiva entre los conocimientos previos y la nueva información y el rendimiento académico

\begin{tabular}{llc}
\hline & Indicador & Rendimiento académico \\
\hline Estrategias para mejorar & Coeficiente de correlación & .648 \\
la integración constructiva & Sig. (bilateral) & .000 \\
$\begin{array}{lc}\text { entre los conocimientos pre- } \\
\text { vios y la nueva información }\end{array}$ & $\mathrm{N}$ & 131 \\
\hline
\end{tabular}

En la tabla 3 se muestra que el coeficiente de correlación es de .648, signo bilateral .000 entre las estrategias para mejorar la integración constructiva entre los conocimientos previos y la nueva información con el rendimiento académico. Estos datos demuestran la relación positiva que se da entre estas dos variables. 
Tabla 4. Prueba de la Correlación de Spearman entre el uso de los organizadores visuales y el rendimiento académico

\begin{tabular}{llc}
\hline & Indicador & Rendimiento académico \\
\hline Uso de los organizadores & Coeficiente de correlación & .592 \\
visuales & Sig. (bilateral) & .000 \\
& $\mathrm{~N}$ & 131 \\
\hline
\end{tabular}

En la tabla 4 se manifiesta que el coeficiente de correlación muestra una cantidad de .592 y la de signo bilateral .000 entre la variable uso de organizadores visuales y rendimiento académico. Estas cantidades nos permiten afirmar que entre ambas variables se manifiesta una relación de carácter positivo y un carácter de relación aceptable.

\section{DISCUSIÓN}

La investigación sobre la relación entre las estrategias de enseñanza y el rendimiento académico en los estudiantes de la especialidad de Primaria y EBI de la Facultad de Ciencias Sociales, Educación y de la Comunicación de la UNASAM reporta que existe relación positiva entre ambas variables. En general, el coeficiente de correlación es positivo debido a que alcanza más del 50\%, el signo bilateral .000 de un total de 131 estudiantes encuestados. A continuación se desarrolla en forma concreta las diferentes estrategias de enseñanza que repercuten en el logro positivo en el rendimiento académico de los estudiantes.

En primer lugar, la prueba de correlación de Spearman entre las estrategias de enseñanza y el rendimiento académico establece un coeficiente de correlación de .671, signo bilateral .000 (tabla 1). Esto implica que la correlación presenta signo positivo, por lo cual la relación entre ambas variables es de tipo directo o positivo. Así mismo, el grado de relación es aceptable entre ambas variables. Este resultado ratifica lo señalado por Díaz-Barriga y Hernández (2010), quienes sostienen que las estrategias de enseñanza son los caminos que se traza el docente para llegar a la meta propuesta; en este caso es el buen rendimiento académico de los estudiantes. Además, los resultados hacen entrever que los métodos interactivos surten mejor efecto ante los métodos tradicionales de enseñanza referidos a los expositivos.

Sobre las decisiones acerca de los métodos a utilizar en el aula son el fruto de la conjunción de varios factores de las concepciones del docente sobre el aprendizaje y su cultura profesional, de sus concepciones sobre las ciencias sociales y de las finalidades educativas que pretende; además también influyen los métodos propios de las disciplinas sociales y algunas consideraciones más contextuales como la complejidad de las tareas, su coste en el aula o el número de estudiantes a los que se debe atender. Por consiguiente, los logros en el rendimiento académico en gran medida son el resultado de la aplicación de diversidad de estrategias metodológicas usadas por el docente.

En segundo lugar, los resultados en cuanto a si los docentes realizan actividades para conocer cuánto saben los estudiantes sobre el tema que van a desarrollar. La prueba de la Correlación de Spearman entre las estrategias para activar y usar los conocimientos previos y el rendimiento académico reporta un coeficiente de .591 y el signo bilateral .000 (tabla 2). Estos resultados permiten establecer que la correlación presenta signo positivo, por lo cual la relación entre ambas variables es directa o positiva. Así mismo, el grado de relación es aceptable entre ambas variables. 
Al respecto, Ausbel (1976) y Miras (1993) refieren que existen estrategias para activar y usar los conocimientos previos y para generar expectativas apropiadas en los alumnos. Las estrategias para las sesiones de aprendizaje se deben emplear al inicio de cada secuencia didáctica, o bien antes de que los estudiantes inicien algún tipo de actividad de indagación, discusión o integración sobre el material de aprendizaje propiamente dicho, sea de manera individual o colaborativa.

Las estrategias que corresponden a este grupo están dirigidas a activar o generar los conocimientos previos en los aprendices. Los aprendizajes que se logran sobre la base de los conocimientos previos son los aprendizajes significativos, entonces son de trascendental importancia los conocimientos previos en la construcción del conocimiento. De ahí la importancia de activar los conocimientos previos pertinentes de los estudiantes utilizando estrategias didácticas apropiadas (estrategias de actividad focal introductoria, discusiones guiadas, actividad generadora de información previa, entre otras), con el fin de retomarlos y relacionarlos en momentos adecuados a la información nueva por aprender que se descubre o construye de manera conjunta con los alumnos (Ausubel, 2002).

En tercer lugar, con relación a la activación de los conocimientos previos que tienen los estudiantes y la conexión con lo nuevo por aprender, la prueba de la Correlación de Spearman, entre las estrategias para mejorar la integración constructiva entre los conocimientos previos y la nueva información y el rendimiento académico establece un coeficiente de correlación .648 y un signo bilateral de .000 (tabla 3). Estos resultados permiten afirmar que la correlación presenta signo positivo, por lo cual la relación entre ambas variables es de tipo directo o positivo. Así mismo, el grado de relación es aceptable entre ambas variables. Este resultado es concordante con la concepción constructivista de la educación. Para dicho fin las estrategias se utilizan antes o durante el proceso instructivo para lograr mejores resultados en el aprendizaje y, por tanto, de rendimiento académico.

Las estrategias están orientadas a ayudar a crear enlaces adecuados entre los conocimientos previos y la información nueva por aprender, asegurando con ello una mayor significatividad de los aprendizajes logrados y un mejor despliegue de la enseñanza, entendida esta desde la óptica de la ayuda ajustada a la ZDP. De acuerdo con Mayer (1984), a este proceso de integración entre lo «previo» y lo «nuevo» se le denomina: «construcción de conexiones externas». Las estrategias típicas de enlace entre lo nuevo y lo previo son las de inspiración ausubeliana: los organizadores previos y las analogías.

En cuarto lugar, con relación al uso de los organizadores visuales y el rendimiento académico, la prueba de correlación de Spearman reporta que el coeficiente de correlación es .592 y el signo bilateral .000 (tabla 4). Como se puede ver, la correlación presenta signo positivo, por lo cual la relación entre ambas variables es de tipo directo o positivo. Así mismo, el grado de relación es aceptable entre ambas variables.

Como se puede apreciar existe una estrecha relación entre ambas variables, pues los recursos didácticos signados en los organizadores visuales u organizadores gráficos son muy efectivos para el logro de los objetivos educativos que se traducen en el buen rendimiento académico.

Los organizadores visuales son representaciones visuales que comunican la estructura lógica del material instruccional que va a aprenderse (Trowbridge y Wandersee, 1998; Armbruster, 1994; West, Farmer y Wolff, 1991; citado por Díaz-Barriga s/f.). Su efectividad ha sido ampliamente comprobada en ambos casos para la mejora de 
los procesos de recuerdo, comprensión y aprendizaje. Como estrategia de enseñanza pueden usarse en cualquier momento del proceso didáctico. Hay una amplia variedad de organizadores y también varios intentos de clasificación desarrollados en la literatura especializada. Sin embargo, los más utilizados por los docentes de la FCSEC que laboran en la especialidad de Primaria y EBI son los mapas conceptuales, una estructura jerarquizada en diferentes niveles de generalidad o inclusividad conceptual; están formados por conceptos, proposiciones y palabras de enlace (Novak, 1998; Novak y Gowin, 1988; Ontoria, 1993).

Podemos decir, entonces, que el empleo de las estrategias didácticas diversas guarda una relación directa con los logros de aprendizaje y el rendimiento académico de los estudiantes. Por consiguiente, a mayor y mejor uso de las estrategias de enseñanza, las estrategias para activar y usar los conocimientos previos, las estrategias para mejorar la integración constructiva entre los conocimientos previos y la nueva información, así como el uso de los organizadores visuales tienen una relación directa con el rendimiento académico de los estudiantes de la especialidad de Primaria y Educación Bilingüe Intercultural de la Facultad de Ciencias Sociales, Educación y de la Comunicación de la Universidad Nacional «Santiago Antúnez de Mayolo».

Finalmente, podemos precisar que en este tema no se incluyó a las TIC's por las razones siguientes: (1) La intención de la investigación fue percibir qué tanto se relacionan dos variables a partir del planteamiento ofrecido por un determinado autor. (2) Dada la naturaleza de la investigación y literatura consultada el uso de las TIC's queda relegado a un segundo plano, lo cual no significa no valorar su trascendencia en el proceso educativo.

\section{CONCLUSIONES}

Existe una relación positiva entre las estrategias de enseñanza y el rendimiento académico de los estudiantes de Primaria y Educación Bilingüe Intercultural de la Facultad de Ciencias Sociales, Educación y de la Comunicación de la UNASAM.

En el semestre académico 2013-II, los estudiantes de Primaria y EBI registran un 85\% de aprobación; con promedio de calificación más alta (14). Los que tienen mejor percepción sobre el uso de las estrategias por los docentes son los de 5to. ciclo hacia adelante.

Se percibe relación positiva entre el uso de los organizadores visuales y el rendimiento académico, dado que los estudiantes registran calificativos más altos en las asignaturas donde los docentes utilizan estrategias visuales.

De igual manera, la correlación positiva entre el uso de las discusiones guiadas, como estrategia de enseñanza, con el rendimiento académico en los estudiantes se evidencia en la aprobación del 85\% de ellos en los cursos de formación general.

Existe una relación directa entre la utilización de los organizadores previos como estrategia de enseñanza con el rendimiento académico en los estudiantes que se percibe en los promedios más altos en las asignaturas de especialidad.

Se evidencia una relación positiva entre el uso de los organizadores visuales -mapa conceptual y semántico- como estrategias de enseñanza y el rendimiento académico en los estudiantes, utilizados por los docentes con formación pedagógica y cursos de capacitación a nivel de maestría y doctorado. 


\section{REFERENCIAS BIBLIOGRÁFICAS}

Ausbel, David. 1976. Psicología educativa. México: Trillas.

Ausbel, David. 2002. Adquisición y retención del conocimiento. Una perspectiva cognitiva. Barcelona: Paidós.

Castellá, Josep; Comelles, Salvador; Cros, Ana y Vila, Monserrat. 2007. Entender (se) en clase. Barcelona: Graó.

Díaz-Barriga, Frida (s/f). Estrategias docentes para un aprendizaje significativo. <http://148.228.165.6/PES/fhs/ANEXO_ESTRATEGIAS/Cuadros\%20 y\%20diagramas.pdf. $>$ [Consulta: 14-07-15].

Díaz-Barriga, Frida y Hernández, Gerardo. 2010. Estrategias docentes para un aprendizaje significativo. Una interpretación constructivista. 3ra. Ed. México: Mc Graw Hill.

Eggen, Paul y Kauchak, Donald. 1999. Estrategias docentes. Enseñanza de contenidos y desarrollo de habilidades del pensamiento. Buenos Aires: FCE.

Mayer, Richard. 1984. 'Aids to text comprehension'. Educational Psychologist, 19 (1), $43-64$.

Mayer, Richard. 2004. Psicología de la educación. Enseñar para un aprendizaje significativo. Vol. 2. Madrid: Pearson.

Mercer, Neil. 2001. Las perspectivas socioculturales y el estudio del discurso en el aula. En César Coll y Derek Edwards (Eds.). Enseñanza, aprendizaje y discurso en el aula. Madrid: Fundación infancia y aprendizaje.

Miras, Mariana. 1993. Un punto de partida para el aprendizaje de nuevos contenidos: los conocimientos previos. En C. Coll et al. El constructivismo en aula. Barcelona: Graó.

Novak, Joseph y Gowin, Bob. 1988. Aprendiendo a aprender. Barcelona: Martínez Roca.

Novak, Joseph. 1998. Conocimiento y aprendizaje. Los mapas conceptuales como herramientas facilitadoras para escuelas y empresas. Madrid: Alianza.

Ontoria, Antonio. 1993. Mapas conceptuales. Una técnica para aprender. Madrid: Narcea.

Quiroz, María. 2001. Aprendiendo en la era digital. Lima: Fondo Editorial de la Universidad de Lima.

Rodríguez, Sebastián; Fita, Eva y Torrado, Mercedes. 2004. «El rendimiento académico en la transición secundaria-universidad». Revista de Educación. Temas actuales de enseñanza, $\mathrm{N}^{\mathrm{o}} 334.391-414$.

Tovar, Julio. 2008. «Modelo metacognitivo como integrador de Estrategias de enseñanza y estrategias de aprendizaje de las Ciencias y su relación con las competencias». Revista Iberoamericana de educación. No 46/7-25. <http://www. rieoei.org/deloslectores/2161Tovarv2.pdf.> [Consulta: 05- 07-15].

Trowbridge, John y Wandersee, James. 1998. «Organizadores gráficos, teoría impulsada». En J.J. Mintzes, J.H. Wandersee, y JD Novak (Eds.). La enseñanza de la ciencia para la comprensión. Academic Press.

Wray, David y Lewis, Maureen. 2005. Aprender a leer y escribir textos de información. 2 da. Edición. Madrid: Morata.

Fecha de recepción: 12 de noviembre 2015

Fecha de aceptación: 07 de diciembre 2015

\section{Correspondencia}

Laura Nivin Vargas

lauranivin@yahoo.es 